\title{
Magnitude of Mistreatment and Associated Factors Among Delivering Mothers in Bishoftu General Hospital, Oromia, Ethiopia
}

\author{
Yared Mekonen Tefera, Sileshi Garoma Abeya *, Sultan Kalu Beggi \\ Department of Public Health, Adama Hospital Medical College, Adama, Ethiopia \\ Email address: \\ kibreyar668@gmail.com (Y. M. Tefera), garomaabe@gmail.com (S. G. Abeya), sultanate2010@gmail.com (S. K. Beggi) \\ ${ }^{*}$ Corresponding author
}

To cite this article:

Yared Mekonen Tefera, Sileshi Garoma Abeya, Sultan Kalu Beggi. Magnitude of Mistreatment and Associated Factors Among Delivering Mothers in Bishoftu General Hospital, Oromia, Ethiopia. International Journal of Neurologic Physical Therapy.

Vol. 5, No. 1, 2019, pp. 13-20. doi: 10.11648/j.ijnpt.20190501.13

Received: May 7, 2019; Accepted: June 10, 2019; Published: June 25, 2019

\begin{abstract}
The recent evidence has demonstrated that globally, many women experience mistreatment during labor and delivery in health facilities. This can pose a significant barrier to women attending facilities for delivery and can contribute to poor birth experience and adverse outcome for the woman and newborn. The objective of this study was to assess the magnitude of mistreatment among delivering mothers and associated factors in Bishoftu general hospital from June December, 2018. An institutional based cross-sectional study design was conducted. A Single population proportion formula was used to select a total of 377 participants. The convenience sampling method was employed to select the study participants and Focused Group Discussion was conducted for qualitative data collection. The collected Data was coded, cleaned, by using Epi. Info and analyzed by SPSS 20. Principally, binary logistic regression model was used to indicate the association between the study variables. The Association between independent and dependent variable were considered significant when the pvalue is less than 0.05 in the final model. Qualitative data from the Focus Group Discussions were analyzed and presented thematically. In this study, $55.8 \%(95 \% \mathrm{CI}$ : $51.2,61.9)$ of the interviewed mothers reported having perceived at least one form of mistreatment. The types of mistreatment during childbirth included physical abuse (21.1\%), non-consented care (27.4\%), non-confidential care $(11.7 \%)$, non-dignified care $(9.7 \%)$, abandonment $(24.8 \%)$, and discrimination (11.4\%). Parity $(\mathrm{AOR}=1.85,95 \% \mathrm{CI}=1.05,3.24)$ and mode of delivery $(\mathrm{AOR}=1.75,95 \% \mathrm{CI}=1.09,2.81)$ were factors significantly associated with mistreatment. This study has shown that the proportion of mistreatment during health facility childbirth in Bishoftu General Hospital was high. Parity and modes of delivery were significantly associated with mistreatment. Efforts to improve quality of maternal care should include greater training and monitoring of providers to ensure respectful treatment of patients.
\end{abstract}

Keywords: Mistreatment, Disrespect and Abuse, Childbirth, Respectful Maternity Care

\section{Introduction}

Increasing access to skilled care during childbirth is a key strategy for reducing maternal and early neonatal mortality and morbidity [1]. Evidence has demonstrated that globally, many women face mistreatment during labor and delivery in health facilities which can pose a significant barrier to woman attending facilities for delivering and other service. This can contribute to poor birth experience and adverse outcome for women and newborns [2]. A woman's experience of care in childbirth is an important determinant of her future decisions related to seeking health care from health facilities because birth experience is individual, unique, and unforgettable [1]. Evidence is mounting that mistreatment may undermine women's trust in the health system and deters them from seeking facility-based care for delivery [3].

World Health Organization (WHO) defines mistreatment as "interactions or facility conditions that local consensus seems to be humiliating or undignified and those interactions or conditions that are experienced as or intended to be humiliating or undignified" [4]. Disrespect and abuse or 
mistreatment related to childbirth was recently introduced and conceptualized in 2010 and 2015, respectively [5-6]. A model on types of mistreatment during facility based childbirth from landscape analysis explored seven categories including, physical abuse; non-consented care; nonconfidential care; non- dignified care; discrimination; abandonment of care and detention in facilities [7].

Indeed, there is no similar and accurate estimates of the prevalence of mistreatment exists and, notably, there is also no operational definition of mistreatment the concept is defined only in its absence [7]. However, a recent mixed method systematic review that was conducted globally showed the proportion of women who reported experiencing any mistreatment during childbirth was $19.5 \%$ with common specific experiences included "non- dignified care (12.9\%) [5]. Also, studies conducted in six European countries found that one in five pregnant women had experienced some form of abuse (e.g. Being degraded, blackmailed, insulted, or abused physically and/or emotionally) when receiving health services over her lifetime; and a history of abuse in health care was associated with the increased fear of birth during pregnancy [8]. In different countries the prevalence of mistreatment was $10 \%$ in Brazil (Pelotas town) [9], 10\% in Tanzania [10], 20\% in Kenya [11], and 36\% in Ethiopia [12].

In various studies, marital and educational statuses of women, types of health facility, monthly income, use of ANC, types of delivery, waiting time, knowledge and attitudes among health care workers, the working environment and the number of staffs were factors significantly associated with the mistreatment of women during childbirth [2, 11-10].

Mistreatment in facility based childbirth constitutes a huge quality of care problem and is often closely associated with poor clinical quality of care and poor patient satisfaction with care [6]. In addition, mistreatment during childbirth can potentially deter women from seeking medical care in the future, leading to severe negative health implications. Mistreatment during childbirth is not a new phenomenon. However, in recent years, it has gained widespread attention across the international community. This issue was formally prompted by human rights reports and today, several studies $[5,11,13]$ confirm that women across the globe frequently mistreated during childbirth [14]. Humanization of childbirth is a unique approach which helps to make childbirth is a positive and satisfying experience for both the woman and her family. This strategy used to empower woman and their care provider by considering humanized values such as the woman's emotional state, their values, beliefs, sense of dignity and autonomy during childbirth [15].

Recently, increased attention is being paid to the reasons why women, who know fully the benefits of facility-based deliveries and who have the means to access a facility, continue to choose home births [16]. Evidence from multiple countries in Sub-Saharan Africa showed that women would prefer to deliver in a facility but choose home delivery not to because of the presence of inadequate, low quality, and disrespectful care in facilities [16]. In Ethiopia, the proportion of childbirths attended by a Skilled Birth
Attendant (SBA) in 2016 was $28 \%$, compared to 50 to $53 \%$ in other Sub-Saharan African countries, especially in East Africa [17]. One of the reason for low rate of childbirth assisted by skilled birth attendants is absence of respectful maternity care and the actual and perceived high mistreatment committed by health providers [18]. But there is inadequate research on the role of mistreatment of women during facility based deliveries in decreasing utilization of maternity services. Currently compassionate and respectful maternity care during labor and delivery considered an important component of healthcare provider quality assurance program. Previous studies only focus on the maternity care users/individual level, but they neglect to consider other contributors of mistreatment like provider related factors. Therefore, this study was conducted to assess the magnitude of mistreatment among delivering mothers and associated factors in Bishoftu general hospital, Oromia, Ethiopia, from September 22, 2018 - February 16, 2019 G.C.

\section{Materials and Methods}

\subsection{Study Area}

This study was conducted in Bishoftu General Hospital in Oromia region in Ethiopia. Bishoftu town is located $47 \mathrm{Kms}$ south east of Addis Ababa (the capital of Ethiopia). In this town, there are nine and three urban and rural sub-cities, respectively. According to Bishoftu town administration health office report, the total population during 2017/2018 was 186,753 of which $95,244(51 \%)$ are females. The numbers of women who are in child bearing age group (1549 ) were 41,328 , of which 3,313 were pregnant and about 4518 received labor and delivery care services during the year 2018 [19].

\subsection{Study Design}

An institution based cross-sectional study design was employed using quantitative and qualitative data collection methods.

\subsection{Populations}

All pregnant women who have got delivery service in Bishoftu General Hospital were the source population, while, all randomly sampled pregnant women who gave birth during the study period were the study population. Mothers who were health professionals and/or working in the study facility and gave birth during the study period were excluded from the study because it was believed that they might be treated with respect by their fellows. Moreover, women with complication during labor and delivery and in the postpartum period were also excluded.

\subsection{Sample Size Determination}

\subsubsection{Quantitative Method}

The single population proportion formula was used with the assumptions of $67.1 \%$ of delivered mothers would face at 
least one form of mistreatment during childbirth [20], 5\% margin of error $(d), 95 \%$ confidence level $\left(Z_{\alpha / 2}\right)$, and with the possible $10 \%$ non-response rate. Based on the following formula;

$$
\begin{gathered}
\mathrm{n}=\frac{(\mathrm{Z} \alpha / 2)^{2} \mathrm{P}(1-\mathrm{P})}{\mathrm{d} 2} \\
\mathrm{n}=\frac{(1.96) 2 * 0.67(0.33)}{(0.05)^{2}} \mathrm{n}=340
\end{gathered}
$$

Since the source population (N) 4, $518 \mathrm{w}$ less than 10,000, the finite population correction formula was used to reduce the sample size:

$$
\begin{aligned}
& \mathrm{n}=\frac{n}{1+\frac{n}{N}} \\
& =\frac{340}{1+\frac{340}{4518}}=316
\end{aligned}
$$

Using the above formula, the estimated sample size of the study was 316 from the recent health facility delivered women. For the possible non-response of $10 \%$ of calculated sample size was adjusted as follows.

$$
\mathrm{n}_{\text {final }}=\frac{316 \times 1}{R}
$$

Where $\mathrm{R}$ is the Response Rate $=90 \%=\frac{316 \times 1}{0.9}=351$

\subsubsection{Qualitative Method}

A total of six Focused Group Discussions was held. Discussants were selected purposefully. Grouping was based on similar character and those having direct or indirect contact with the study participants. Two groups were formed from sub-city administration and community leaders. Two groups from woman's affaires focal person, town health and education bureaus. The rest two groups were from women having the birth experience of one or more times in the health facilities. The discussions were held in quiet places.

\subsection{Sampling Procedure}

For the quantitative study, systematic random sampling was to recruit eligible respondents. Accordingly, every other mothers were approached to be interviewed during the discharge time until the required sample size was met.

\subsection{Data Collections}

A structured and pre-tested interviewer administered questionnaire was used to collect the data. The questionnaire was adapted from Maternal and Child Health Integrated Program (MCHIP) to assess mistreatment [21]. The questionnaire was modified according to the study contexts to answer the objective. The questionnaire and discussion guides were prepared in English and translated into Amharic and Afan Oromo (regional working language) by different language experts. Six midwives were recruited as a data collector and supervisor from outside the study facility based on the criteria of having respectful maternity care training and able to speak and write the regional working language
(Afan Oromo). Three days training was given for the study team on the objective of the study and data collection procedure. Mothers were interviewed after they have completed everything on exit. Focused Group Discussions were held by one social worker having Master degree and one note taker with that of the principal investigator.

\subsection{Data Processing and Analysis}

Data entry was done using Epi-info version 7 and exported to statistical Package for Social Sciences (SPSS) version 20 for analysis. The frequencies of different categories of mistreatment were reported in the tables. A binary and multiple logistic regression analysis was employed to explain the study variables. In the bivariate analysis, variables with a P-value of $\leq 0.25$ were a candidate for a multivariable regression model. In the final model, the p-value is less than 0.05 were considered significant association between the study variables. The multicollinearity effect between independent variables was checked using tolerance and variance inflation factor. The Qualitative data from Focused Group Discussions were analyzed and presented thematically.

\subsection{Operational Definitions}

Mistreatment: mistreatment during childbirth can represent a violation of women's reproductive rights [5]. A woman who reported and observed at least one incident from the asked and observed verification criteria in the category was labeled as Mistreated [21].

Disrespect: is rude conduct and usually considered to indicate a lack of respect or violation of maternity care rights of mothers; at least one of the criteria is missed, it classified as disrespected [21].

Physical abuse: physical force or abrasive behavior with the woman, including slapping or hitting and touches measured using seven criteria, a woman who answers yes to at list one criteria, then she was considered as being abused at the time of labor and delivery [22].

Non-confidential care: lack of confidentiality and lack of privacy during maternal care measured using two criteria, a women who answers yes to at list one of the criteria, then she was considered as being abused at the time of labor and delivery [22].

Non-consented care: absence of informed consent, or patient communication, forced procedure, measured using seven criteria, a woman who answers yes to at least one criteria, then she was considered as being abused at the time of labor and delivery [22].

Non-dignified care: lack of dignity, respect and intentionally humiliating, scolding, or shouting at patient's value and for women; measured using two criterion, a woman who answers yes to at list one of the criteria then she was considered as being abused at the time of labor and delivery [22].

Discrimination: luck of equitable care measured using three criteria a woman who answers yes to at least one criteria, then she was considered as being abused at the time 
of labor and delivery.

Abandonment or denial of care: lack of right to timely health care and to the highest Attainable level of health, measured using two criteria, a woman who answers yes to at list one of the criteria then she was considered as being abused at the time of labor and delivery [22].

\subsection{Ethical Considerations}

The study was conducted after obtaining administrative permission and ethical clearance from the Adama Hospital Medical College, Research Ethics Committee (REC) and Institution of Review Board (IRB) of the Oromia Regional Health Bureau. Verbal consents were obtained from each participant to participate in the study. Anonymity and confidentiality were strictly done. Participants were told of the possibility of withdrawing or leaving the study at any time if they don't want and that would not have any impact on their access to services.

\section{Results}

\subsection{Socio-Demographic Characteristics}

Three hundred seventy seven delivering mothers were planned to be included in the study and it was possible to interview 351 making a response rate of $93 \%$. The majority of respondents 112 (31.9\%) were between the age group of 26-29 years with the mean $( \pm \mathrm{SD})$ age of $27.53( \pm 5.31)$ years. About $247(70.4 \%)$ of the study participant were urban residents. Most, 276 (78.4\%) of them were married. Concerning their educational background and occupation, 127 (36.2\%) of the study participant were attending secondary school, and 182 $(51.9 \%)$ were employed, respectively. The median (IQR) monthly income was calculated to be 1000 ETB and 189 $(53.8 \%)$ of the respondents earning below the median income level (<1000 ETB) (Table 1).

Table 1. Socio-demographic characteristics of the respondent, Bishoftu, April 2019.

\begin{tabular}{llll}
\hline Variables & Response Category & Number & Percent \\
\hline \multirow{4}{*}{ Age in years } & $18-21$ & 49 & 14 \\
& $22-25$ & 79 & 22.5 \\
& $26-29$ & 112 & 31.9 \\
Residence & $\geq 30$ & 111 & 31.6 \\
& Urban & 247 & 70.4 \\
Marital status & Rural & 104 & 29.6 \\
& Married & 276 & 78.6 \\
& Unmarried & 75 & 21.4 \\
Level of Education & Technic and above & 114 & 32.5 \\
& Secondary & 127 & 36.2 \\
& Primary & 59 & 16.8 \\
& Illiterate & 51 & 14.5 \\
Occupation & Employed & 182 & 51.9 \\
\hline
\end{tabular}

\subsection{Obstetrics Characteristics}

Of the total respondents, $231(65.8 \%)$ were reported to have a planned pregnancy and nearly all 345 (98.3\%) mothers had a history of ANC follow-up. Among them, about
$231(65.8 \%)$ of the respondent had a history of four and more visits for ANC service. About 211 (60.1\%) of respondents had previous history of childbirth, of which 125 (35.6\%) mothers previously gave birth in the same facility. The mean $( \pm \mathrm{SD})$ duration of labor was $8.66( \pm 2.99)$ hours. About half $176(50.1 \%)$ of mother's delivered within eight hours of labor time. About 186 (50.3\%) of mother gave birth through spontaneous vaginal delivery. The calculated Median client waiting time is five and $296(84.3 \%)$ of mothers have got service within five minutes (Table 2).

Table 2. Obstetrics characteristics of mothers, Bishoftu, Ethiopia, April 2019.

\begin{tabular}{llll}
\hline Variables & Response Category & Frequency & Percent \\
\hline Planned & Yes & 231 & 65.8 \\
pregnancy & No & 120 & 34.2 \\
ANC attended & Yes & 345 & 98.3 \\
Number of ANC & No & 6 & 1.7 \\
follow-up & $\geq 4$ & 213 & 60.7 \\
& $<4$ & 132 & 37.6 \\
Parity & Multipara & 202 & 57.5 \\
& Primipara & 149 & 42.5 \\
Duration of labor & $<8$ hours & 176 & 50.1 \\
& $>12$ hours & 121 & 34.5 \\
Mode of delivery & SVD & 54 & 15.4 \\
Client waiting time to & $<5$ & 186 & 53 \\
get service (Minute) & $\geq 5$ & 165 & 47 \\
\hline
\end{tabular}

\subsection{Mistreatment During Childbirth}

One hundred ninety six $(55.8 \%),(95 \% \mathrm{CI}: 51.2,61.9)$ of the respondents reported experiencing at least one form of Mistreatment during childbirth. Accordingly, 74 (21.1\%) of women reported to have physical harm or ill treatment. For $65(18.5 \%)$ of the respondents Episiotomy was done without anesthesia. This was supported by qualitative study finding that showed suturing without antipain during labour and delivery were commonly practiced at the maternity care unit at the health facility.

"During the time of delivery with cut around the birth canal, the health care provider used and repaired it without painkillers, it was so painful I don't want to remember that time, I felt very bad". (FGD, mother 34 yrs).

Indeed, discussants had mixed opinions about health care services provided to the clients without consent. Some reported that, they were asked for their consent before any procedure, while others reported as the health workers knew what was right for them.

The other most commonly experienced mistreatment was the prohibition of mothers informed consent, and choice/preferences that accounted for 96 (27.4\%). The commonly violated criterion under this domain was providers didn't ask verbal consent during instrumental delivery for 33 (9.4\%) of them. Some participants who delivered at health facilities consistently reported that consent was not obtained before any procedures or examinations were carried out in addition to this in $83(23.6 \%)$ of the respondents who didn't have information about the procedure. 
A 39 year mother from women's group who had delivered at hospital previously supports this idea and said that "... The health care provider used a metal instrument to take the baby out and told me to react when I have, he didn't ask me my permission and also he didn't tell me".

The third commonly reported types of mistreatment were non-confidential care which accounts 41 (11.4\%). Commonly violated criterion under this domain was health care providers didn't Uses screen or covering appropriate to protect mother's privacy $29(8.3 \%)$. This is quite different from the health facility observation report, that presence of curtain and mobile screen in between the bed, In addition to this $34(9.7 \%)$ of women reported Non-dignified care Majority $29(8.3 \%)$ of respondent reported under this domain were health providers shouted at during labour and delivery. Related to this some of the participants agreed that health workers differed in character, with some being courteous while some were not.

However, the participant in the FDG reported saying "my friend's sister was admitted in labor ward to give birth after a while the labor pain is increasing and the woman started to shout like"erdugn, memmote new" meaning "please help me, I am going to die" and during that time the nurse become frustrated and impatient and started shouting on her" (birth companion, 35 year)

On the other hand, from total respondent 87 (24.8\%) of women were abandoned by a health care provider when they needed help. Under this domain 66 (18.8\%) of women were left un-attended after delivery. The other category of mistreatment was discrimination, $40(11.4 \%)$ of woman reported that they feel discriminated during childbirth (Figure 1).

This study showed that lack of infrastructure during maternity care was one of determinate cause that leads to mistreatment of the maternity care user. Almost all maternity care users and community leader who participated on FGD reported that there is inadequate beds, and delivery coach in the health facility. One mother in FDG this idea and reported that:

"I gave birth in the hospital in which the number of client flow is very high. There was only three delivery coach and I gave birth in the waiting room. All mothers and their birth companion observed me and my privacy was not respected, I felt depressed" (mother, 36 years)

In the discussion, the individual behavior of health care professionals was well stressed, in which some of them are tempered. For example, if a midwife gives instructions to the mother and the mother fails to comply promptly she/he may scold or beat her due to anger. One participant in FGD wondered by saying:

"Such a problem may be caused by someone's character. Actually a nurse can't do such bad things to the patient while she is required to help. But it depends on the nurses behavior, although there are other annoyance done when the mother is asked doing something and do not cooperate. It is good for nurses to tolerate them because they are in pain. However, other nurses can't and they end up scolding and beating mothers regardless of the pain they are experiencing due to anger" (birth companion, 30years).

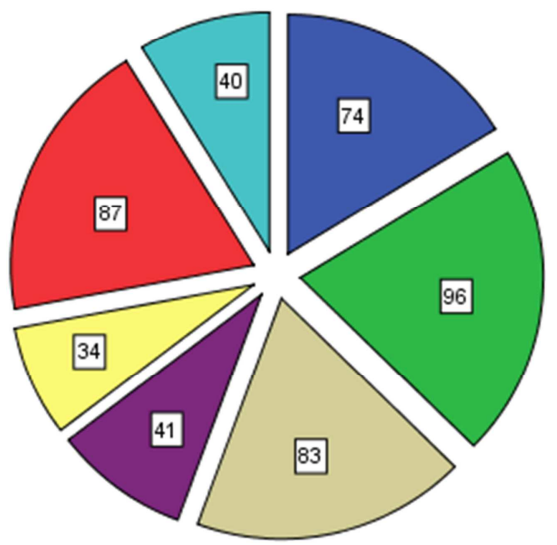

$\square$ physical abuse Non-consented care $\square$ information $\square$ Non-confidential care $\square$ Non-dignified care $\square$ Abandonment $\square$ Discrimination

Figure 1. Prevalence of Mistreatment women during childbirth, Bishoftu, April 2019.

\subsection{Factors Associated with Mistreatment Among Delivering Mother}

At the binary logistic regression analysis age, residence, marital status, planned pregnancy, number of ANC attended, parity duration of labor and mode of delivery was found to be a candidate for multiple logistic regression analysis $(\mathrm{p}<$ 0.25). However, in the final model parity and mode of delivery were significantly associated with mistreatment ( $p$ value $<0.05)$. Accordingly, primipara mothers were more nearly two (AOR, 1.85; 95\% CI: 1.05, 3.24) times more likely to be mistreated compared to multipara mothers. Moreover, women not having spontaneous vaginal delivery were 1.75 times (AOR, 1.75; 95\% CI, 1.09, 2.81) more likely to be mistreated compared to their counterparts (Table 3 ).

Table 3. Factors associated with mistreatment during labor and delivery among mothers who have given birth, Bishoftu, April 2019.

\begin{tabular}{|c|c|c|c|c|c|}
\hline \multirow{2}{*}{ Variables } & \multirow{2}{*}{ Response Category } & \multicolumn{2}{|c|}{ Mistreatment } & \multirow{2}{*}{$\operatorname{COR}(95 \% \mathrm{CI})$} & \multirow{2}{*}{$\operatorname{AOR}(95 \% C I)$} \\
\hline & & Yes $(\%)$ & No $(\%)$ & & \\
\hline \multirow{4}{*}{ Age in years } & $18-21$ & $40(81.6)$ & $9(18.4)$ & $4.69(2.08 .10 .58)^{* * *}$ & $0.39(0.16,1.01)$ \\
\hline & $22-25$ & $43(54.4)$ & $36(45.6)$ & $1.26(0.71,2.25)$ & $0.45(0.18,1.13)$ \\
\hline & $26-29$ & $59(52.7)$ & $53(47.3)$ & $1.18(0.69,1.99)$ & $0.47(0.18,1.23)$ \\
\hline & 30 and above & $54(48.6)$ & $57(51.4)$ & $1: 00$ & $1: 00$ \\
\hline \multirow{2}{*}{ Marital Status } & Unmarried & $55(73.3)$ & $20(26.7)$ & $2.63(1.50,4.63)^{* * *}$ & $1.48(0.79,2.80)$ \\
\hline & Married & $141(51.1)$ & $135(48.9)$ & $1: 00$ & $1: 00$ \\
\hline Residence & Rural & $68(65.4)$ & $36(34.6)$ & $1.76(1.09,2.82)^{*}$ & $1.64(0.93,2.89)$ \\
\hline
\end{tabular}




\begin{tabular}{|c|c|c|c|c|c|}
\hline \multirow{3}{*}{ Variables } & \multirow{2}{*}{ Response Category } & \multicolumn{2}{|c|}{ Mistreatment } & \multirow{2}{*}{ COR $(95 \% \mathrm{CI})$} & \multirow{2}{*}{ AOR $(95 \% C I)$} \\
\hline & & Yes (\%) & No (\%) & & \\
\hline & Urban & $128(51.8)$ & $119(48.2)$ & $1: 00$ & $1: 00$ \\
\hline \multirow{2}{*}{ Planned pregnancy } & No & $77(64.2)$ & $43(35.8)$ & $1.76(1.09,2.82)^{*}$ & $1.52(0.89,2.58)$ \\
\hline & Yes & $119(51.5)$ & $112(48.5)$ & $1: 00$ & $1: 00$ \\
\hline \multirow{2}{*}{ Number of ANC } & Less than four & $86(62.3)$ & $52(37.7)$ & $1.55(1.00,2.39)^{*}$ & $0.79(0.46,1.38)$ \\
\hline & Four and more & $110(51.6)$ & 103(48.4) & $1: 00$ & $1: 00$ \\
\hline \multirow{2}{*}{ Parity } & \multirow{2}{*}{ Primipara Multipara } & $100(67.1)$ & $49(32.9)$ & $2.25(1.45,3.50)^{* * *}$ & $1.85(1.05,3.24)^{*}$ \\
\hline & & $96(47.5)$ & $106(52.5)$ & $1: 00$ & $1: 00$ \\
\hline \multirow{3}{*}{ Duration of labor } & $>12$ hours & $42(77.8)$ & $12(22.2)$ & $3.50(1.73,7.09)^{* * *}$ & $1.83(0.82,4.12)$ \\
\hline & $8-12$ hours & $66(54.5)$ & $55(45.5)$ & $1.20(0.75,1.90)$ & $0.83(0.49,1.42)$ \\
\hline & $<8$ hours & $88(50)$ & $88(50)$ & $1: 00$ & $1: 00$ \\
\hline \multirow{2}{*}{ Mode of delivery } & Other than SVD & $109(66.1)$ & $56(33.9)$ & $2.22(1.42,3.41)^{* * * *}$ & $1.75(1.09,2.81)$ \\
\hline & SVD & $87(46.8)$ & $99(53.2)$ & $1: 00$ & $1: 00$ \\
\hline
\end{tabular}

NB: COR-Crud Odds Ratio

AOR- Adjusted odds Ratio

SVD- spontaneous vaginal delivery

$*_{-} \mathrm{P}<0.05$ **. $\mathrm{P}<0.01$ ***. $\mathrm{P}<0.001$

\section{Discussions}

The study revealed a significant proportion $(55.8 \%$, $95 \%$ CI:51.2, 61.9) of mothers experienced at least one form of mistreatment while giving birth in the facility. Most of the events were categorized as disrespectful and abusive maternity care as defined by Bowser and Hill (2010). However, the prevalence of the current study is slightly lower than the study conducted in Bahirdar, Ethiopia on level of disrespect and abuse of women and associated factors during facility based childbirth [20], and higher than the studies in northern Ethiopia (22\%) [23]. This discrepancy might be due to the small sample size of the previous study and it might also be due to the differences in the study period and study settings.

In the current study, physical abuse $(21.1 \%)$ is commonly experienced and the component of mistreatment. It is consistent with other previous study in Addis Ababa with respectful and non-abusive maternity care during childbirth in hospital and health center showed $23.7 \%$ of respondents experienced of physical abuse.

In this study, the other type of mistreatment experienced were non-consented care that includes non-informed consent, and absence of choice/preference with the prevalence of $27.4 \%$. This is much higher than study conducted at the northern part of Ethiopia on the mother's experience of mistreatment with a prevalence of $7.7 \%$ [23]. But, it is lower than the study conducted in Addis (94.8\%) of which about $48 \%$ of mother were not asked for their consent or permission prior to any procedure. Basically, it is critical that healthcare providers should offer the women adequate explanation and obtain informed consent before administering treatment and performing any procedure. Lack of information given to women before or during procedures for women during childbirth was the common practices associated with mistreatment in sub-Saharan Africa [2, 24-25]. The other category of mistreatment of women experienced during facility based childbirth in this study was non-confidentiality care that accounted for $11.7 \%$. This finding is different from the studies conducted in urban Tanzanian showed $2 \%$ and $8.5 \%$ in Kenya [5, 24].

From FGD with maternity care users and community leaders perceived that high caseloads and limited staff in maternity care resulting in poor care, overworked providers, and work-related stresses that might negatively influence their attitudes towards their work. Normalization of mistreatment, lack of good attitude of health care provider towards mothers and his/her work were also another identified factors for mistreatment during labour and delivery. This study finding was almost similar to the study conducted in Kenya on prevalence mistreatment during facility based child birth [24].

From this study non-dignified care (11.4\%) and discrimination (11.4\%) where the last category of mistreatment which is much lower compared to a study conducted on mothers' experience of disrespect and abuse during maternity care in northern Ethiopia (53\%) and (39\%), respectively [23].

From the results of Focus Group Discusions participants of maternity care users, community leaders and health care providers which showed that the contributing factors for the mistreatment of women during facility based child birth was lack of infrastructures in the health facility. The frustration of healthcare workers might be experienced as a result of problems of heavy workload and poor infrastructure, poor motivation/job satisfaction and mother's uncooperative behavior which might jeopardize both the mother's and unborn baby's lives [26].

In this study, the mother who gave birth for the first time were more likely to be mistreated. This might be because of intolerable pain during labour and delivery due to un-laxed pelvic organs compared to mothers who had multiple deliveries. Also, in this study, those mothers who gave birth by destructive deliveries and cesarean section were more likely to be mistreated. These types of deliveries were happening due to prolonged labour and mal-presentations causing exhaustion and pain for the mother and noncompliance to the health care workers command.

The limitation of the Study, information bias is inevitable, 
as the study relied on self reported data, hence there was no objective measure of the prevalence. The participants were interviewed in the health facility settings, though there was an attempt to reduce any uncertainties providing good establishment of rapport, providing privacy and assuring their confidentiality. Also, there were a courtesy bias in which some husbands refused to allow their wives to participate in the study. However, the study team worked to dispel these fears by reassuring potential participants by convincing the study was related to childbirth, and would not put them at risk of to get service.

\section{Conclusions}

This study has shown that the proportion of mistreatment during health facility childbirth in Bishoftu General Hospital is high. Physical abuse and dis-respected care are the commonly practice events. Parity and mode of delivery had significant association with mistreatment among delivering mothers by their delivery attendants in the hospital. As mistreatment of women by the care provider during childbirth is a pervasive health and human rights violation, efforts to improve quality of maternal care should include greater training and monitoring of providers to ensure respectful treatment of patients.

\section{Acknowledgements}

We are very much grateful to Adama Hospital Medical College for funding the study. Our thanks also go to all the study teams and the participants for their cooperation during the processes of the data collection. All the staffs of Bishoftu General Hospital are acknowledged for their permission and cooperation.

\section{References}

[1] Mehretie Y. Experience of Facility Based Childbirth in Rural Ethiopia An Exploratory Study of Women's Perspective. journal of pregnancy, November, 2017.

[2] Asefa A, Bekele D. Status of respectful and non-abusive care during facility-based childbirth in a hospital and health centers in Addis Ababa, Ethiopia. Reprod Health. 2015 16;12:33. doi: 10.1186/s12978-015-0024-9

[3] Ratcliffe HL, Sando D, Lyatuu GW, Emil F, MwanyikaSando M, Chalamilla G, Langer A, McDonald KP. Mitigating disrespect and abuse during childbirth in Tanzania an exploratory study of the effects of two facility-based interventions in a large public hospital. Reprod Health. 2016, 18;13 (1):79. doi: 10.1186/s12978-016-0187-z

[4] Freedman LP, Kruk ME. Disrespect and abuse of women in childbirth: challenging the global quality and accountability agendas. The Lancet, 2014. 384 (9948): p. e42-e44.

[5] David S, Tamil K, Goodluck L, Hannah R, Kathleen MD, Mary MS, Faida E, Guerino Chalamilla, M, Ana L. Disrespect and Abuse During Childbirth in Tanzania: Are Women Living With HIV More Vulnerable? December 1, 2014.
[6] Bowser D, Hill K, Exploring evidence for disrespect and abuse in facility-based childbirth: report of a landscape analysis, in USAID-TRAction Project, Washington, DC. 2010.

[7] Rima J. Respectful Maternity Care: The Universal Rights of Childbearing Women (Poster), 2011. Available at http/ $\mathrm{http}: / /$ www.healthpolicyproject.com/index.cfm?ID=publicatio ns\&get=pubID\&pubID=44.

[8] Lukasse M, SchrollA-M, Karro H, Schei B, Steingrimsdottir T, VanParys A-S, Elsa LR, Ann T. Prevalence ofexperienced abuse in healthcare andassociated obstetric characteristics in sixEuropean countries. Acta Obstet GynecolScand 2015; 94: $508-517$

[9] Mesenburg MA. Disrespect and abuse of women during the process of childbirth in the 2015 Pelotas birth cohort. 2015.

[10] Estimates by WHO, UNICEF, UNFPA, World Bank Group and the United Nations Population Division. Trends in maternal mortality: 1990 to 2015. Available at http/ https://www.afro.who.int/sites/default/files/2017-05/trends-inmaternal-mortality-1990-to-2015.pdf

[11] Aguiar JM, d'Oliveira AF, Schraiber LB. Institutional violence, medical authority, and power relations in maternity hospitals from the perspective of health workers. Cadernos de saude publica. 2013;29 (11):2287-96.

[12] Mary F, Brigid Mc. Respectful Maternity Care and the Media: A Guide for NGOs and Advocates was copyedited by John Engels and designed by Gwendolyn Stinger, 2019. Available a: https://www.whiteribbonalliance.org/wpcontent/uploads/2019/02/RMC_Media_Guide.pdf

[13] Sudhinaraset M, Treleaven E, Melo J, Singh K, DiamondSmith N. Women's status and experiences of mistreatment during childbirth in Uttar Pradesh: a mixed methods study using cultural health capital theory. BMC Pregnancy Childbirth. 2016 28;16 (1):332

[14] Hameed W,. Avan BI. Women's experiences of mistreatment during childbirth: A comparative view of home- and facilitybased births in Pakistan. PLoS One, 2018. 13 (3): p. e0194601.

[15] Ayele, S., level of disrespect and abuse in maternity care among facility based maternity care users, debre markose, east gojjam, ethiopia. Nov 2016. Available at: http://etd.aau.edu.et/browse?type=advisor\&sort_by $=1 \&$ order $=$ ASC\&rpp $=20 \&$ etal $=-$

$1 \&$ value $=\mathrm{ABIY}+\mathrm{SEIFU}+\% 28 \mathrm{MPH} \% 29 \&$ starts_with $=0$

[16] Bohren MA, Vogel JP, Tunçalp Ö, Fawole B, Titiloye MA, Olutayo AO, Ogunlade M, Oyeniran AA, Osunsan OR, Metiboba L, Idris HA, Alu FE, Oladapo OT, Gülmezoglu AM, Hindin MJ. Mistreatment of women during childbirth in Abuja, Nigeria: a qualitative study on perceptions and experiences of women and healthcare providers. Reprod Health. 2017 Jan 17;14 (1):9. doi: 10.1186/s12978-016-0265-2.

[17] Bohren MA, Vogel JP, Hunter EC, Lutsiv O, Makh SK, Souza JP, Carolina A, Fernando S, Alex L, Özge T, Dena J, Olufemi T, Rajat K, Michelle J, Metin G. The Mistreatment of Women during Childbirth in Health Facilities Globally: A MixedMethods Systematic Review. PLoS Med 12 (6): e10018

[18] Turan JM, Bukusi EA, Cohen CR, Sande J, Miller S. Effects of HIV/AIDS on maternity care providers in Kenya. J Obstet Gynecol Neonatal Nurs. 2008 Sep-Oct;37 (5):588-95. doi: 10.1111/j.1552-6909.2008.00281. x. 
[19] Annual Plan and Achievment of the Year 2015/16. 2016. East Shoa Zone health department, Oromia, Ethiopia, 2017.

[20] Biresaw Wasihun1, L. D., Nadia Worede2, Teklemariam Gultie1,, Prevalence of Disrespect and abuse of women during child birth and associated factors in Bahir Dar town, Ethiopia 2017.

[21] Wassihun B, Deribe L, Worede N, Gultie T. Prevalence of Disrespect and abuse of women during child birth and associated factors in Bahir Dar town, Ethiopia. Epidemiol Health. 2018 1;40:e2018029. doi: 10.4178/epih.e2018029. eCollection 2018.

[22] Organization WH, U., Trends in maternal mortality: 1990 to 2013: estimates by WHO, UNICEF, UNFPA, The World Bank and the United Nations Population Division:. executive summary., 2014.
[23] Mengistu WG, Araya AM, Yemane B. Mothers' experience of disrespect and abuse during maternity care in northern Ethiopia. Global Health Action, 2018 (11), 1465215.

[24] Abuya T, Warren CE, Miller N, Njuki R, Ndwiga C, Maranga A, Faith M, Anne N, Ben B. Exploring the Prevalence of Disrespect and Abuse during Childbirth in Kenya. PLoS ONE 10 (4): e0123606. doi:10.1371/ journal. pone.0123606.

[25] Ephrem DS, Eva B, Hannah G, Hone BF, Firew A, Tsigereda BB, Maria MW, Aelaf EK, Sintayehu AW, Young-MK, Akker T, Jelle S. Respectful maternity care in Ethiopian public health facilities. 2017.

[26] Wendy H, Maya G. "Being treated like a human being": Attitudes and behaviours of reproductive and maternal health care providers August 2012. Available at: https://www.burnet.edu.au/system/asset/file/1408/Holmes_et al_attitudes_review_sept 2 final.pdf 